\title{
ON THE YIELD CONDITION FOR ANISOTROPIC MATERIALS*
}

\author{
BY \\ G. F. SMITH \\ Yale University
}

1. Introduction. The yield condition for an incompressible perfectly plastic material may be characterized by a function

$$
F(\mathbf{s})=0,
$$

where $\mathbf{s}\left(=\left\|s_{i j}\right\|\right)$ is the stress deviation matrix which is of course symmetric and subject to the condition that

$$
s_{11}+s_{22}+s_{33}=0 .
$$

The yield function $F$ is required to be invariant under the group of transformations $\{\mathbf{T}\}=\mathbf{T}_{1}, \mathbf{T}_{2}, \cdots, \mathbf{T}_{p}$ associated with the symmetry properties of the material, i.e.,

$$
F\left(\mathbf{T}_{i} \mathbf{s}_{i}^{-1}\right)=F(\mathbf{s}) \quad(i=1, \cdots, p) .
$$

In this paper a set of quantities $J_{1}, \cdots, J_{n}$ will be determined for each of the crystal classes of the hexagonal and cubic crystal systems such that each of the $J_{1}, \cdots, J_{n}$ is invariant under the group of transformations $\{\mathbf{T}\}$ associated with the given crystal class and such that every single-valued function of the matrix $\mathbf{s}$ which is invariant under the group $\{\mathbf{T}\}$, and hence the yield function $F(\mathbf{s})$, will be expressible as a singlevalued function of the $J_{1}, \cdots, J_{n}$. The quantities $J_{1}, \cdots, J_{n}$ will be called a functional basis.

The restrictions imposed on $F$ by the equations (1.3) have been investigated by von Mises [1] for certain of the hexagonal and cubic crystal classes. The argument employed in [1] may be stated as follows: Since there are only five independent variables among the $s_{i j}$ due to the condition (1.2), there are at most five functionally independent invariants $N_{1}, \cdots, N_{5}$ (say) and all other invariants must be expressible as a function of the $N_{1}, \cdots, N_{5}$. It will be shown in Secs. 3 and 4 that for both of the cases considered in [1], the quantities $N_{1}, \cdots, N_{5}$ given there do not form a set of five functionally independent invariants and the results are consequently not valid. It is further observed that for the crystal classes of the hexagonal and cubic systems, an arbitrary invariant $F$ is not in general expressible as a single-valued function of five invariants $N_{1}, \cdots, N_{5}$ even if they are functionally independent.

2. Functional bases. A functional basis for functions of a symmetric matrix $\mathbf{s}$ which are invariant under the group of transformations $\{\mathbf{T}\}=\left\{\mathbf{T}_{1}=\mathbf{I}, \mathbf{T}_{2}, \cdots, \mathbf{T}_{p}\right\}$ is defined as a set of functions $J_{1}(\mathbf{s}), \cdots, J_{n}(\mathbf{s})$, each of which is invariant under the group $\{\mathbf{T}\}$, such that there is exactly one set of solutions

$$
\{\mathbf{s}\}=\left\{\mathbf{T}_{1} \mathbf{s T}_{1}^{-1}, \mathbf{T}_{2} \mathbf{s T}_{2}^{-1}, \cdots, \mathbf{T}_{p} \mathbf{s T}_{p}^{-1}\right\}
$$

to the equations

$$
J_{1}(\mathbf{s})=a_{1}, \quad J_{2}(\mathbf{s})=a_{2}, \cdots, J_{n}(\mathbf{s})=a_{n}
$$

* Received October 3, 1961; revised manuscript received January 4, 1962. 
for all consistent sets of values of $a_{1}, \cdots, a_{n}$. The solutions (2.1) may be said to form a set of solutions equivalent with respect to $\{\mathbf{T}\}$. Since, by definition, any single-valued function of $\mathbf{s}$ invariant under $\{\mathbf{T}\}$ will assume the same value for each of the solutions (2.1), it is clear that the values of $J_{1}, \cdots, J_{n}$ determine uniquely the values of all single-valued functions invariant under $\{\mathbf{T}\}$. Thus, any single-valued function invariant under $\{\mathbf{T}\}$, and hence the yield function $F$, is expressible as a single-valued function of the elements of the functional basis.

It is known [2] that an integrity basis* will also form a functional basis if the group $\{\mathbf{T}\}$ is finite. Integrity bases for functions of a symmetric tensor invariant under the group of transformations appropriate to each of the crystal classes have been determined by Smith and Rivlin [3] and these sets of quantities would serve as functional bases. However, it is possible for the cases considered here to determine smaller sets of invariants which also form functional bases. Thus, if $J_{1}, \cdots, J_{m}$ are elements of the integrity basis, the equations

$$
J_{1}(\mathrm{~s})=a_{1}, \cdots, J_{m}(\mathrm{~s})=a_{m}
$$

will specify a single set of solutions for $\mathbf{s}$ of the form (2.1) which are equivalent with respect to the group $\{\mathbf{T}\}$ considered. If the values of $J_{n+1}, \cdots, J_{m}$ (say) are specified in all circumstances by the values of $J_{1}, \cdots, J_{n}$, then it is clear that the invariants $J_{1}, \cdots, J_{n}$ will still suffice to specify a single set of solutions equivalent with respect to $\{\mathbf{T}\}$. Hence, the quantities $J_{1}, \cdots, J_{n}$ will also form a functional basis.

3. The cubic system. In this and the following section, functional bases will be determined for each of the crystal classes of the cubic and hexagonal crystal systems. The nomenclature adopted for the various crystal classes is that employed by Dana and Hurlbut [4]. The symmetry properties of these crystal classes may be described in terms of the transformations I, C, $\mathbf{R}_{1}, \mathbf{R}_{2}, \mathbf{R}_{3}, \mathbf{D}_{1}, \mathbf{D}_{2}, \mathbf{D}_{3}, \mathbf{T}_{1}, \mathbf{T}_{2}, \mathbf{T}_{3}, \mathbf{M}_{1}, \mathbf{M}_{2}$, $S_{1}, S_{2}$ where $I$ is the identity transformation; $C$ is the central inversion transformation; $\mathbf{R}_{1}, \mathbf{R}_{2}, \mathbf{R}_{3}, \mathbf{T}_{1}, \mathbf{T}_{2}, \mathbf{T}_{3}$ are reflection transformations and $\mathbf{D}_{1}, \mathbf{D}_{2}, \mathbf{D}_{3}, \mathbf{M}_{1}, \mathbf{M}_{2}, \mathbf{S}_{1}, \mathbf{S}_{2}$ are rotation transformations. Explicit expressions for the matrices defining these transformations are given in [3]. The group of symmetry transformations associated with each crystal class together with the set of transformations of the matrix $\mathbf{s}$ induced by them will be listed in the sequel. The integrity basis given in [3] corresponding to the crystal class considered will also be listed and it will then be shown that the values of certain of the elements of the integrity basis are determined in all cases by the remaining elements $J_{1}, \cdots, J_{n}$ (say). The quantities $J_{1}, \cdots, J_{n}$ will then form a functional basis for the crystal class considered.

(i) the tetartoidal (I, $\left.\mathbf{D}_{1}, \mathbf{D}_{\mathbf{2}}, \mathbf{D}_{3}\right) \cdot\left(\mathbf{I}, \mathbf{M}_{\mathbf{1}}, \mathbf{M}_{2}\right.$ ) and diploidal (I, $\mathbf{C}, \mathbf{R}_{\mathbf{1}}, \mathbf{R}_{\mathbf{2}}, \mathbf{R}_{\mathbf{3}}$, $\left.\mathbf{D}_{1}, \mathbf{D}_{2}, \mathbf{D}_{3}\right) \cdot\left(\mathbf{I}, \mathbf{M}_{1}, \mathbf{M}_{2}\right)$ crystal classes. It is seen [3] that for these crystal classes the requirement (1.3) is to be satisfied for the twelve sets of $\overline{\mathbf{s}}=\mathbf{T s T}^{-1}$ given by

$$
\begin{aligned}
& \left(\bar{s}_{11}, \bar{s}_{22}, \bar{s}_{33}, \bar{s}_{12}, \bar{s}_{23}, \bar{s}_{31}\right)=\left(s_{i i}, s_{i j}, s_{k k}, s_{i j}, s_{j k}, s_{k i}\right), \\
& \left(s_{i i}, s_{j i}, s_{k k}, s_{i j},-s_{i k},-s_{k i}\right), \quad\left(s_{i i}, s_{i j}, s_{k k},-s_{i i}, s_{i k},-s_{k i}\right), \\
& \left(s_{i i}, s_{i j}, s_{k k},-s_{i j},-s_{i k}, s_{k i}\right)
\end{aligned}
$$

where $(i, j, k)=(1,2,3),(2,3,1),(3,1,2)$. In this section, the quantity $\sum s_{i_{1} j_{2}} s_{i, i_{2}} \cdots s_{i_{n} i_{n}}$

*An integrity basis is a set of polynomials, each invariant under $\{\mathrm{T}\}$, such that any polynomial function invariant under $\{\mathbf{T}\}$ is expressible as a polynomial in the elements of the integrity basis. 
will denote the sum of the three quantities obtained by permuting the subscripts in the summand cyclically. For example,

$$
\sum s_{11}\left(s_{31}^{2}-s_{12}^{2}\right)=s_{11}\left(s_{31}^{2}-s_{12}^{2}\right)+s_{22}\left(s_{12}^{2}-s_{23}^{2}\right)+s_{33}\left(s_{23}^{2}-s_{31}^{2}\right) .
$$

With this notation, an integrity basis for these crystal classes is formed [3] by the quantities

$$
\begin{aligned}
J_{1} & =\sum s_{11}, \quad J_{2}=\sum s_{11} s_{22}, \quad J_{3}=s_{11} s_{22} s_{33}, \\
J_{4} & =\sum s_{23}^{2}, \quad J_{5}=\sum s_{23}^{2} s_{31}^{2}, \quad J_{6}=s_{23} s_{31} s_{12}, \\
J_{7} & =\sum s_{11}\left(s_{31}^{2}+s_{12}^{2}\right), \quad J_{8}=\sum s_{11} s_{31}^{2} s_{12}^{2}, \quad J_{9}=\sum s_{11}\left(s_{31}^{2}-s_{12}^{2}\right), \\
J_{10} & =\sum s_{11} s_{22}\left(s_{11}-s_{22}\right), \quad J_{11}=\sum s_{23}^{2} s_{31}^{2}\left(s_{23}^{2}-s_{31}^{2}\right)
\end{aligned}
$$

and

$K_{1}=\sum s_{23}^{2} s_{22} s_{33}, \quad K_{2}=\sum s_{11} s_{22}\left(s_{31}^{2}-s_{23}^{2}\right), \quad K_{3}=\sum s_{23}^{2} s_{31}^{2}\left(s_{22}-s_{11}\right)$.

It may be readily verified that

$$
\begin{gathered}
K_{1}\left(J_{4}^{2}-3 J_{5}\right)=3 J_{6}^{2}\left(3 J_{2}-J_{1}^{2}\right)+2 J_{1} J_{4} J_{8}-3 J_{7} J_{8}+J_{2} J_{4}^{3} \\
\quad+J_{4} J_{5}\left(J_{1}^{2}-4 J_{2}\right)-J_{1} J_{4}^{2} J_{7}+J_{4} J_{7}^{2}, \\
2 K_{2}\left(J_{1}^{2}-3 J_{2}\right)=\left(J_{1} J_{2}-9 J_{3}\right) J_{9}-2 J_{1} J_{4} J_{10}+3 J_{7} J_{10}, \\
2 K_{3}\left(J_{4}^{2}-3 J_{5}\right)=\left(9 J_{6}^{2}-J_{4} J_{5}\right) J_{9}-2 J_{1} J_{4} J_{11}+3 J_{7} J_{11} .
\end{gathered}
$$

The values of $K_{1}$ and $K_{3}$ are then specified by the values of the quantities (3.2) unless

$$
2\left(J_{4}^{2}-3 J_{5}\right)=\left(s_{23}^{2}-s_{31}^{2}\right)^{2}+\left(s_{31}^{2}-s_{12}^{2}\right)^{2}+\left(s_{12}^{2}-s_{23}^{2}\right)^{2}=0 .
$$

This can occur only if $s_{23}^{2}=s_{31}^{2}=s_{12}^{2}$ in which case the values of $K_{1}$ and $K_{3}$ are given by $\frac{1}{3} J_{2} J_{4}$ and 0 respectively. Similarly, the value of $K_{2}$ is specified by the values of the quantities (3.2) unless

$$
2\left(J_{1}^{2}-3 J_{2}\right)=\left(s_{11}-s_{22}\right)^{2}+\left(s_{22}-s_{33}\right)^{2}+\left(s_{33}-s_{11}\right)^{2}=0
$$

in which case $K_{2}=0$. Hence, the values of $K_{1}, K_{2}$ and $K_{3}$ are specified in all cases by the values of the quantities (3.2). Consequently, the quantities (3.2) form a functional basis for the tetartoidal and diploidal classes. Since $J_{1}=0$ from (1.2), any yield function is expressible as a single valued function of the quantities $J_{2}, \cdots, J_{11}$ defined by (3.2).

(ii) the hextetrahedral $\left(\mathbf{I}, \mathbf{D}_{1}, \mathbf{D}_{2}, \mathbf{D}_{3}\right) \cdot\left(\mathbf{I}, \mathbf{M}_{1}, \mathbf{M}_{2}, \mathbf{T}_{1}, \mathbf{T}_{2}, \mathbf{T}_{3}\right.$ ), gyroidal $\left(\mathbf{I}, \mathbf{D}_{1}, \mathbf{D}_{2}, \mathbf{D}_{3}\right) \cdot\left(\mathbf{I}, \mathbf{M}_{1}, \mathbf{M}_{2}\right),\left(\mathbf{C}, \mathbf{R}_{1}, \mathbf{R}_{2}, \mathbf{R}_{3}\right)$. $\left(\mathbf{T}_{1}, \mathbf{T}_{2}, \mathbf{T}_{3}\right)$ and hexoctahedral (I, C, $\left.\mathbf{R}_{1}, \mathbf{R}_{2}, \mathbf{R}_{3}, \mathbf{D}_{1}, \mathbf{D}_{2}, \mathbf{D}_{3}\right) \cdot\left(\mathbf{I}, \mathbf{M}_{1}, \mathbf{M}_{2}, \mathbf{T}_{1}, \mathbf{T}_{2}, \mathbf{T}_{3}\right.$ ) crystal classes. It is seen [3] that for these crystal classes the requirement (1.3) is to be satisfied for the twenty-four sets of $\overline{\mathbf{s}}$ given by $(3.1)$ with $(i, j, k)=(1,2,3),(2,3,1),(3,1,2),(1,3,2),(3,2,1),(2,1,3)$. An integrity basis for these crystal classes is formed [3] by the quantities $J_{i}(i=1, \cdots, 8)$ and $K_{1}$ defined by (3.2) and (3.3). From the previous section,.it is seen that the value of $K_{1}$ is completely specified in all cases by the values of $J_{1}, \cdots, J_{8}$. Hence, the quantities $J_{1}, \cdots, J_{8}$ defined by (3.2) form a functional basis. Since $J_{1}=0$ from (1.2), any yield function $F$ for the hextetrahedral, gyroidal and hexoctahedral crystal classes is expressible as a single-valued function of the quantities $J_{2}, \cdots, J_{8}$ defined by (3.2). 
Mises [1] states that for these crystal classes any yield function is expressible in terms of the quantities

$$
\begin{array}{ll}
N_{1}=\sum\left(s_{11}-s_{22}\right)^{2}, & N_{2}=\sum s_{12}^{2}, \quad N_{3}=s_{12} s_{23} s_{31}, \\
N_{4}=\sum\left(s_{11}-s_{22}\right)^{3}, & N_{5}=\sum\left(s_{11}+s_{22}-2 s_{33}\right) s_{12}^{2} .
\end{array}
$$

It is noted $N_{4}$ changes sign under the transformation

$$
\left(\bar{s}_{11}, \bar{s}_{22}, \bar{s}_{33}, \bar{s}_{12}, \bar{s}_{23}, \bar{s}_{31}\right)=\left(s_{22}, s_{11}, s_{33}, s_{12}, s_{31}, s_{23}\right)
$$

and consequently is not invariant under the group of transformations associated with this crystal class. Thus, an arbitrary function of the $N_{1}, \cdots, N_{5}$ is not necessarily a possible yield function.

4. The hexagonal system. (i) the trigonal-pyramidal $\left(\mathbf{I}, \mathbf{S}_{1}, \mathbf{S}_{2}\right)$ and rhombohedral (I, C). (I, $\mathbf{S}_{1}, \mathbf{S}_{2}$ ) crystal classes. It is seen [3] that for these crystal classes the requirement (1.3) is to be satisfied for the three sets of $\overline{\mathbf{s}}$ given by

$$
\begin{array}{r}
\left(\bar{s}_{11}, \bar{s}_{22}, \bar{s}_{33}, \bar{s}_{12}, \bar{s}_{23}, \bar{s}_{31}\right)=\left(s_{11}, s_{22}, s_{33}, s_{12}, s_{23}, s_{31}\right), \\
\left(\frac{1}{4} s_{11}+\frac{3}{4} s_{22} \mp \frac{3^{1 / 2}}{2} s_{12}, \frac{3}{4} s_{11}+\frac{1}{4} s_{22} \pm \frac{3^{1 / 2}}{2} s_{12}, s_{33},-\frac{1}{2} s_{12} \pm \frac{3^{1 / 2}}{4}\left(s_{11}-s_{22}\right)\right. \\
\left.-\frac{1}{2} s_{23} \mp \frac{3^{1 / 2}}{2} s_{31},-\frac{1}{2} s_{31} \pm \frac{3^{1 / 2}}{2} s_{23}\right) .
\end{array}
$$

An integrity basis for these crystals classes is formed [3] by the quantities

$$
\begin{aligned}
J_{1} & =s_{11}+s_{22}, \quad J_{2}=s_{11} s_{22}-s_{12}^{2}, \quad J_{3}=s_{11}\left[\left(s_{11}+3 s_{22}\right)^{2}-12 s_{12}^{2}\right], \\
J_{4} & =s_{33}, \quad J_{5}=s_{31}^{2}+s_{23}^{2}, \quad J_{6}=s_{23}\left(s_{23}^{2}-3 s_{31}^{2}\right), \\
J_{7} & =\left(s_{11}-s_{22}\right) s_{23}+2 s_{12} s_{31}, \quad J_{8}=s_{22} s_{31}^{2}+s_{11} s_{23}^{2}-2 s_{23} s_{31} s_{12} \\
J_{9} & =\left(s_{11}-s_{22}\right) s_{31}-2 s_{12} s_{23}, \quad J_{10}=3 s_{12}\left(s_{11}-s_{22}\right)^{2}-4 s_{12}^{3} \\
J_{11} & =s_{31}\left(s_{31}^{2}-3 s_{23}^{2}\right)
\end{aligned}
$$

and

$$
\begin{aligned}
& K_{1}=s_{31}\left[\left(s_{11}+s_{22}\right)^{2}+4\left(s_{12}^{2}-s_{22}^{2}\right)\right]-8 s_{11} s_{12} s_{23}, \\
& K_{2}=s_{23}\left[\left(s_{11}+s_{22}\right)^{2}+4\left(s_{12}^{2}-s_{22}^{2}\right)\right]+8 s_{11} s_{12} s_{31}, \\
& K_{3}=\left(s_{11}-s_{22}\right) s_{23} s_{31}+s_{12}\left(s_{23}^{2}-s_{31}^{2}\right) .
\end{aligned}
$$

It may be readily verified that

$$
\begin{aligned}
& J_{5} K_{1}=\left(J_{1}^{2}-4 J_{2}\right) J_{11}+4 J_{8} J_{9}, \\
& J_{5} K_{2}=\left(J_{1}^{2}-4 J_{2}\right) J_{6}+4\left(J_{1} J_{5}-J_{8}\right) J_{7}, \\
& 2 J_{5} K_{3}=-J_{7} J_{11}-J_{6} J_{9} .
\end{aligned}
$$

The values of $K_{1}, K_{2}$ and $K_{3}$ are then specified by the values of $J_{1}, \cdots, J_{11}$ unless $J_{5}=s_{31}^{2}+s_{23}^{2}=0$. This can occur only if $s_{31}=s_{23}=0$ in which case $K_{1}=K_{2}=K_{3}=0$. Hence, the values of $K_{1}, K_{2}$ and $K_{3}$ are specified in all cases by the values of $J_{1}, \cdots, J_{11}$. Consequently, the quantities $J_{1}, \cdots, J_{11}$ defined by (4.2) form a functional basis for the trigonal-pyramidal and rhombohedral crystal classes. Since $J_{1}=-J_{4}$ from (1.2), 
any yield function $F$ is expressible as a single-valued function of the quantities $J_{2}, \cdots, J_{11}$ defined by (4.2).

(ii) the ditrigonal-pyramidal $\left(\mathbf{I}, \mathbf{R}_{1}\right) \cdot\left(\mathbf{I}, \mathbf{S}_{1}, \mathbf{S}_{2}\right)$, trigonal-trapezohedral $\left(\mathbf{I}, \mathbf{D}_{1}\right) \cdot$ $\left(\mathbf{I}, \mathbf{S}_{1}, \mathbf{S}_{2}\right.$ ) and hexagonal-scalenohedral $\left(\mathbf{I}, \mathbf{C}, \mathbf{R}_{1}, \mathbf{D}_{1}\right) \cdot\left(\mathbf{I}, \mathbf{S}_{1}, \mathbf{S}_{2}\right.$ ) crystal classes. It is seen [3] that for these crystal classes the requirement (1.3) is to be satisfied for the six sets of $\overline{\mathbf{s}}$ given by (4.1) and

$$
\begin{gathered}
\left(\bar{s}_{11}, \bar{s}_{22}, \bar{s}_{33}, \bar{s}_{12}, \bar{s}_{23}, \bar{s}_{31}\right)=\left(s_{11}, s_{22}, s_{33},-s_{12}, s_{23},-s_{31}\right), \\
\left(\frac{1}{4} s_{11}+\frac{3}{4} s_{22} \mp \frac{3^{1 / 2}}{2} s_{12}, \frac{3}{4} s_{11}+\frac{1}{4} s_{22} \pm \frac{3^{1 / 2}}{2} s_{12}, s_{33}, \frac{1}{2} s_{12} \mp \frac{3^{1 / 2}}{4}\left(s_{11}-s_{22}\right)\right. \\
\left.-\frac{1}{2} s_{23} \mp \frac{3^{1 / 2}}{2} s_{31}, \frac{1}{2} s_{31} \mp \frac{3^{1 / 2}}{2} s_{23}\right) .
\end{gathered}
$$

An integrity basis for these crystal classes is formed [3] by the quantities $J_{1}, \cdots, J_{\mathbf{8}}$ and $K_{2}$ defined by (4.2) and (4.3). From the previous section, it is seen that the value of $K_{2}$ is completely specified in all cases by the values of $J_{1}, \cdots, J_{8}$. Hence, the quantities $J_{1}, \cdots, J_{8}$ defined by (4.2) form a functional basis for the ditrigonal-pyramidal, trigonal-trapezohedral and hexagonal-scalenohedral crystal classes. Since $J_{1}=-J_{4}$ from (1.2), any yield function $F$ is expressible as a single-valued function of the quantities $J_{2}, \cdots, J_{8}$ defined by (4.2).

(iii) the trigonal-dipyramidal $\left(\mathbf{I}, \mathbf{R}_{3}\right) \cdot\left(\mathbf{I}, \mathbf{S}_{1}, \mathbf{S}_{2}\right)$, hexagonal-pyramidal $\left(\mathbf{I}, \mathbf{D}_{3}\right)$. $\left(\mathbf{I}, \mathbf{S}_{1}, \mathbf{S}_{2}\right.$ ) and hexagonal-dipyramidal $\left(\mathbf{I}, \mathbf{C}, \mathbf{R}_{3}, \mathbf{D}_{3}\right) \cdot\left(\mathbf{I}, \mathbf{S}_{1}, \mathbf{S}_{2}\right.$ ) crystal classes. It is seen [3] that for these crystal classes the requirement (1.3) is to be satisfied for the six sets of $\bar{s}$ given by (4.1) and

$$
\begin{array}{r}
\left(\bar{s}_{11}, \bar{s}_{22}, \bar{s}_{33}, \bar{s}_{12}, \bar{s}_{23}, \bar{s}_{31}\right)=\left(s_{11}, s_{22}, s_{33}, s_{12},-s_{23},-s_{31}\right) \\
\left(\frac{1}{4} s_{11}+\frac{3}{4} s_{22} \mp \frac{3^{1 / 2}}{2} s_{12}, \frac{3}{4} s_{11}+\frac{1}{4} s_{22} \pm \frac{3^{1 / 2}}{2} s_{12}, s_{33},-\frac{1}{2} s_{12} \pm \frac{3^{1 / 2}}{4}\left(s_{11}-s_{22}\right)\right. \\
\left.\frac{1}{2} s_{23} \pm \frac{3^{1 / 2}}{2} s_{31}, \frac{1}{2} s_{31} \mp \frac{3^{1 / 2}}{2} s_{23}\right)
\end{array}
$$

An integrity basis for these crystal classes is formed [3] by the quantities

$$
\begin{aligned}
J_{1} & =s_{11}+s_{22}, \quad J_{2}=s_{11} s_{22}-s_{12}^{2}, \quad J_{3}=s_{11}\left[\left(s_{11}+3 s_{22}\right)^{2}-12 s_{12}^{2}\right], \\
J_{4} & =s_{31}^{2}+s_{23}^{2}, \quad J_{5}=s_{33}, \quad J_{6}=s_{31}^{2}\left(s_{31}^{2}-3 s_{23}^{2}\right)^{2}, \\
J_{7} & =s_{11} s_{23}^{2}+s_{22} s_{31}^{2}-2 s_{23} s_{31} s_{12}, \\
J_{8} & =s_{11}\left(s_{31}^{4}+3 s_{23}^{4}\right)+2 s_{22} s_{31}^{2}\left(s_{31}^{2}+3 s_{23}^{2}\right)-8 s_{12} s_{23} s_{31}^{3}, \\
J_{9} & =s_{12}\left(s_{31}^{2}-s_{23}^{2}\right)+\left(s_{22}-s_{11}\right) s_{31} s_{23}, \\
J_{10} & =3 s_{12}\left(s_{11}-s_{22}\right)^{2}-4 s_{12}^{3}, \quad J_{11}=s_{31} s_{23}\left[3\left(s_{31}^{2}-s_{23}^{2}\right)^{2}-4 s_{31}^{2} s_{23}^{2}\right]
\end{aligned}
$$

and

$$
\begin{aligned}
& K_{1}=s_{31}^{2}\left[\left(s_{11}+s_{22}\right)^{2}-4\left(s_{22}^{2}-s_{12}^{2}\right)\right]-2 s_{11}\left[\left(s_{11}+3 s_{22}\right)\left(s_{31}^{2}+s_{23}^{2}\right)-4 s_{23} s_{31} s_{12}\right], \\
& K_{2}=s_{23} s_{31}\left[\left(s_{11}+s_{22}\right)^{2}-4\left(s_{22}^{2}-s_{12}^{2}\right)\right]+4 s_{11} s_{12}\left(s_{23}^{2}-s_{31}^{2}\right), \\
& K_{3}=s_{12}\left[\left(s_{31}^{2}+s_{23}^{2}\right)^{2}+4 s_{23}^{2}\left(s_{31}^{2}-s_{23}^{2}\right)\right]-4 s_{31}^{3} s_{23}\left(s_{11}-s_{22}\right) .
\end{aligned}
$$


It may be readily verified that

$$
\begin{aligned}
J_{4}^{2} K_{1} & =\left(J_{1}^{2}-4 J_{2}\right) J_{6}-2 J_{1} J_{4} J_{8}-8 J_{4} J_{7}^{2}+4 J_{7} J_{8}, \\
J_{4}^{2} K_{3} & =\left(3 J_{4}^{3}-2 J_{6}\right) J_{9}-J_{1} J_{4} J_{11}+2 J_{7} J_{11}, \\
\left(J_{1}^{2}-4 J_{2}\right) K_{2} & =\left(2 J_{3}-3 J_{1}^{3}-4 J_{1} J_{2}\right) J_{9}-J_{1} J_{4} J_{10}+2 J_{7} J_{10} .
\end{aligned}
$$

The values of $K_{1}$ and $K_{3}$ are then specified by the values of $J_{1}, \cdots, J_{13}$ unless $J_{4}=s_{31}^{2}+s_{23}^{2}=0$. This can only occur if $s_{31}=s_{23}=0$ in which case $K_{1}=K_{3}=0$. Similarly, the value of $K_{2}$ is specified by the values of $J_{1}, \cdots, J_{11}$ unless

$$
J_{1}^{2}-4 J_{2}=\left(s_{11}-s_{22}\right)^{2}+4 s_{12}^{2}=0 .
$$

This can occur only if $s_{11}=s_{22}$ and $s_{12}=0$ in which case $K_{2}=0$. Hence, the values of $K_{1}, K_{2}$ and $K_{3}$ are specified in all cases by the values of $J_{1}, \cdots, J_{11}$ and consequently the quantities $J_{1}, \cdots, J_{11}$ defined by (4.6) form a functional basis for the trigonaldipyramidal, hexagonal-pyramidal and hexagonal-dipyramidal crystal classes. Since $J_{1}=-J_{5}$ from (1.2), any yield function is expressible as a single-valued function of the quantities $J_{2}, \cdots, J_{11}$ defined by (4.6).

Mises [1] states that, for these crystal classes, any yield function $F$ is expressible in terms of the quantities

$$
\begin{aligned}
& N_{1}=s_{11}+s_{22}-2 s_{33}, \quad N_{2}=\left(s_{11}-s_{33}\right)^{2}+\left(s_{22}-s_{33}\right)^{2}+2 s_{12}^{2}, \\
& N_{3}=\left(s_{11}-s_{22}\right)^{2}+4 s_{12}^{2}, \quad N_{4}=s_{23}^{2}+s_{31}^{2}, \\
& N_{5}=3 s_{23} s_{31}\left(s_{23}^{2}+s_{31}^{2}\right)^{2}-16 s_{23}^{3} s_{31}^{3} .
\end{aligned}
$$

It is readily verified that $N_{3}=2 N_{2}-N_{1}^{2}$ and hence only four of the $N_{1}, \cdots, N_{5}$ are functionally independent invariants. Since an invariant which is functionally independent of the $N_{1}, \cdots, N_{5}$ is readily determined, e.g. $J_{3}$, it is clear that not every yield function is expressible as a function of the $N_{1}, \cdots, N_{5}$.

(iv) the ditrigonal-dipyramidal $\left(\mathbf{I}, \mathbf{R}_{\mathbf{1}}, \mathbf{R}_{\mathbf{3}}, \mathbf{D}_{\mathbf{2}}\right) \cdot\left(\mathbf{I}, \mathbf{S}_{\mathbf{1}}, \mathbf{S}_{2}\right)$, dihexagonal-pyramidal (I, $\left.\mathbf{R}_{1}, \mathbf{R}_{2}, \mathbf{D}_{3}\right) \cdot\left(\mathrm{I}, \mathbf{S}_{1}, \mathbf{S}_{2}\right)$, hexagonal-trapezohedral $\left(\mathbf{I}, \mathbf{D}_{1}, \mathbf{D}_{2}, \mathbf{D}_{3}\right) \cdot\left(\mathrm{I}, S_{1}, \mathbf{S}_{2}\right)$ and dihexagonal-dipyramidal (I, C, $\left.\mathbf{R}_{1}, \mathbf{R}_{2}, \mathbf{R}_{3}, \mathbf{D}_{1}, \mathbf{D}_{2}, \mathbf{D}_{3}\right) \cdot\left(\mathbf{I}, \mathbf{S}_{1}, \mathbf{S}_{2}\right.$ ) crystal classes. For these crystal classes the requirement (1.3) is to be satisfied [3] for the twelve sets of $\bar{s}$ given by (4.1), (4.4), (4.5) and

$$
\begin{array}{r}
\left(\bar{s}_{11}, \bar{s}_{22}, \bar{s}_{33}, \bar{s}_{12}, \bar{s}_{23}, \bar{s}_{31}\right)=\left(s_{11}, s_{22}, s_{33},-s_{12},-s_{23}, s_{31}\right), \\
\left(\frac{1}{4} s_{11}+\frac{3}{4} s_{22} \mp \frac{3^{1 / 2}}{2} s_{12}, \frac{3}{4} s_{11}+\frac{1}{4} s_{22} \pm\right. \\
\frac{3^{1 / 2}}{2} s_{12}, s_{33}, \frac{1}{2} s_{12} \mp \frac{3^{1 / 2}}{4}\left(s_{11}-s_{22}\right), \\
\left.\frac{1}{2} s_{23} \pm \frac{3^{1 / 2}}{2} s_{31},-\frac{1}{2} s_{31} \pm \frac{3^{1 / 2}}{2} s_{23}\right)
\end{array}
$$

An integrity basis is formed [3] by the quantities $J_{1}, \cdots, J_{8}$ and $K_{1}$ defined by (4.6) and (4.7). From Sec. 4 (iii), it is seen that the value of $K_{1}$ is specified in all cases by the values of $J_{1}, \cdots, J_{8}$. Hence, the quantities $J_{1}, \cdots, J_{8}$ defined by (4.6) form a functional basis for the ditrigonal-dipyramidal, dihexagonal-pyramidal, hexagonal-trapezohedral and dihexagonal-dipyramidal crystal classes. Since $J_{1}=-J_{5}$ from (1.2), any yield function is expressible as a single-valued function of the quantities $J_{2}, \cdots, J_{8}$ defined by (4.6). 
Acknowledgment. The author wishes to acknowledge the support of the National Science Foundation.

\section{REFERENCES}

1. R. v. Mises, Mechanik der plastischen Formänderung von Kristallen, Z. angew. Math. Mech. 8, 161-185 (1928)

2. A. C. Pipkin and R. S. Rivlin, The formulation of constitutive equations in continuum physics, Brown Univ. Report No. DA 4531/4 (1958)

3. G. F. Smith and R. S. Rivlin, The strain-energy function for anisotropic elastic materials, Trans. Amer. Math. Soc. 88, 175-193 (1958)

4. J. D. Dana and C. S. Hurlbut, Dana's Textbook of Mineralogy, New York, John Wiley and Sons, 1952 